\title{
Structure of Pareto Solutions of Generalized Polyhedral-Valued Vector Optimization Problems in Banach Spaces
}

\author{
Qinghai He ${ }^{1}$ and Weili Kong ${ }^{2}$ \\ ${ }^{1}$ Department of Mathematics, Yunnan University, Kunming 650091, China \\ ${ }^{2}$ Teacher Education Institute, Qujing Normal University, Qujing 655011, China \\ Correspondence should be addressed to Qinghai He; heqh@ynu.edu.cn
}

Received 3 July 2013; Accepted 6 November 2013

Academic Editor: Marek Wisla

Copyright (c) 2013 Q. He and W. Kong. This is an open access article distributed under the Creative Commons Attribution License, which permits unrestricted use, distribution, and reproduction in any medium, provided the original work is properly cited.

\begin{abstract}
In general Banach spaces, we consider a vector optimization problem (SVOP) in which the objective is a set-valued mapping whose graph is the union of finitely many polyhedra or the union of finitely many generalized polyhedra. Dropping the compactness assumption, we establish some results on structure of the weak Pareto solution set, Pareto solution set, weak Pareto optimal value set, and Pareto optimal value set of (SVOP) and on connectedness of Pareto solution set and Pareto optimal value set of (SVOP). In particular, we improved and generalize, Arrow, Barankin, and Blackwell's classical results in Euclidean spaces and Zheng and Yang's results in general Banach spaces.
\end{abstract}

\section{Introduction}

Let $X$ and $Y$ be Banach spaces, $\Gamma$ a closed convex subset of $X$, $C$ a closed convex cone of $Y, b \in Y$, and $T: X \rightarrow Y$ a linear function. Consider the following optimization problem:

$$
C-\min \{T(x)+b\} \quad \text { subject to } x \in \Gamma \text {. }
$$

$(\mathrm{LMOP})$

Linear multiobjective optimization problem (LMOP) has been extensively studied and applied to various decisionmaking problems in economics, management science, and engineering (see [1-9] and references therein). One of the important topics in vector optimization is the study of the structure of Pareto solution sets. In 1953, Arrow et al. [2] studied the structure of the Pareto solution set, weak Pareto solution set, Pareto optimal value set, and weak Pareto optimal value set for a linear vector optimization problem in Euclidean spaces. In particular, let $X=\mathbb{R}^{m}, Y=\mathbb{R}^{n}$, and $C=\mathbb{R}_{+}^{n}$. If $\Gamma$ is a polyhedron of $R^{m}$ and the Pareto solution set $S$ of (LMOP) is nonempty, then (i) $S$ and the weak Pareto solution set $S_{w}$ of (LMOP) are the unions of finitely many polyhedra of $R^{m}$; (ii) the Pareto optimal value set $V$ and the weak Pareto optimal value set $V_{w}$ are the unions of finitely many polyhedra of $R^{n}$. This theorem is well known as ABB theorem. Since the family of all piecewise linear functions is much larger than that of all linear functions and there exists a wide class of functions that can be approximated by piecewise linear functions, it is of value to study piecewise linear multiobjective optimization (cf. [10-12]). Recently, In Banach spaces setting, Zheng and Yang [11] generalized ABB theorem to the case when the objective is a piecewise linear single-valued mapping. Note that the graph of a piecewise linear function is the union of finitely many polyhedra. Zheng [13] considered the following vector optimization problem:

$$
C-\min F(x) \quad \text { subject to } x \in \Gamma \text {, }
$$

where $F: X \rightrightarrows Y$ is a multifunction whose graph is the union of finitely many convex polyhedra and $\Gamma$ is a polyhedron of $X$. The following results of the structure of (weak) Pareto solution sets were obtained.

Theorem $\mathrm{Z} 1$ (see [13]). Let $X$ and $Y$ be Banach spaces, $\Gamma$ the union of finitely many polyhedra of $X$, and $F: \Gamma \rightrightarrows Y$ a multifunction whose graph $\mathrm{Gr}(F)$ is the union of finitely many convex polyhedra of $X \times Y$. Suppose that the ordering cone $C \subset Y$ is closed, convex, pointed, and has a weakly compact base. If $F(\Gamma)+C$ is convex, then the Pareto solution set $S$ and the Pareto optimal value set $V$ of (SVOP) are the unions of finitely many polyhedra of $X$ and $Y$, respectively. 
Theorem $\mathbf{Z} 2$ (see [13]). Let $X$ and $Y$ be Banach spaces, $\Gamma$ the union of finitely many polyhedra of $X$, and $F: \Gamma \rightrightarrows Y a$ multifunction whose graph $\operatorname{Gr}(F)$ is the union of finitely many convex polyhedra of $X \times Y$. Suppose that the ordering cone $C \subset Y$ is closed, convex, and pointed. If the interior int $(C)$ of $C$ is nonempty, then the weak Pareto solution set $S_{w}$ and the Pareto optimal value set $V_{w}$ of (SVOP) are the unions of finitely many polyhedra of $X$ and $Y$, respectively.

A polyhedron is defined [12-15] as the intersection of finitely many closed half-spaces. Polyhedra exist in many contexts such as linear and quadratic programs, game theory, statistical decision theory, and mathematical biology as well. For more details on the theory of polyhedra, we refer the reader to $[12,14]$ and the references therein. A generalized polyhedron (G-polyhedron or semiclosed polyhedron) in $[13,15]$ is defined as the intersection of finitely many closed and/or open half-spaces. A G-polyhedron can be regarded as an extension of a polyhedron. It has nice properties analogous to a polyhedron; see $[13,15]$ and the references therein. It is necessarily noted that the graph $\operatorname{Gr}(F)$ of a multifunction $F$ is closed when it is the union of finitely many polyhedra, while the graph $\operatorname{Gr}(F)$ is not necessarily closed when it is the union of finitely many G-polyhedra. In the case that $F$ is single valued, the former implies that $F$ is a piecewise continuous linear function but the latter does not imply that $F$ is necessarily a piecewise continuous linear function. Gpolyhedra are important in piecewise linear programs as well as polyhedra. For example, Fang et al. [15] proved that (weak) Pareto solution set of a piecewise linear multicriteria program with possible discontinuity is a union of finitely many Gpolyhedra.

One of our main aims in this work is to investigate the structure of the (weak) Pareto solution set and the (weak) Pareto optimal set of (SVOP) whose graph $\operatorname{Gr}(F)$ is the union of finitely many G-polyhedra.

Another topic in linear optimization problems is to study the connectedness of (weak) Pareto solution sets. Many authors researched this issue; see $[2,11,13,16-20]$ and the references therein. Arrow et al. [2] proved that the (weak) Pareto solution set $\left(S_{w}\right) S$ and the (weak) Pareto optimal value set $\left(V_{w}\right) V$ of (LMOP) are pathwise connected, respectively, when $X=R^{m}, Y=R^{n}$, and $C=R_{+}^{n}$. Recently, Zheng and Yang [11] proved that the weak Pareto set $S_{w}$ and the weak Pareto optimal value set $V_{w}$ of (LMOP) are pathwise connected, respectively, when the ordering cone $C$ has a nonempty interior and the mapping $T(\cdot)+b$ is $C$-convex. Zheng [13] proved that the Pareto set $S$ and the Pareto optimal value set $V$ of (SVOP) are pathwise connected, respectively, when the ordering cone $C$ is a pointed, closed, convex cone with a weakly compact base and $F$ is a $C$-convex multifunction whose graph is the union of finitely many convex polyhedra.

The other of our main aims is to study the connectedness of the Pareto set $S$ and the Pareto optimal value set $V$ of (SVOP) without the assumption of the ordering cone $C$ having a weakly compact base but with that of the cone $C$ being polyhedral.

\section{Preliminaries}

Let $X$ and $Y$ be Banach spaces and let $C \subset Y$ be a convex cone. We say that $C$ is pointed if $C \cap-C=\{0\}$. In this case, one can define a partial order $\leq_{C}$ in $Y$ as follows: for $y_{1}, y_{2} \in$ $Y, y_{1} \leq_{C} y_{2}$ if and only if $y_{2}-y_{1} \in C$. Let int $(C)$ denote the interior of $C$. When $\operatorname{int}(C) \neq \emptyset$, by $y_{1}<_{C} y_{2}$, we mean that $y_{2}-$ $y_{1} \in \operatorname{int}(C)$. Let $Y^{*}$ denote the dual space of $Y$ and $C^{+}$the dual cone of $C$, defined by

$$
C^{+}:=\left\{c^{*} \in Y^{*}:\left\langle c^{*}, c\right\rangle \geq 0, \forall c \in C\right\} .
$$

We denote by $C^{+1}$ the set of all strictly positive continuous linear functionals; that is,

$$
C^{+1}:=\left\{c^{*} \in Y^{*}:\left\langle c^{*}, c\right\rangle>0, \forall c \in C \backslash\{0\}\right\} .
$$

We say a convex subset $\Theta$ of $C$ is a base of $C$ if it satisfies that

$$
C=\left\{t \theta: t \in \mathbb{R}_{+}, \theta \in \Theta\right\}, \quad 0 \notin \mathrm{cl}(\Theta),
$$

where $\mathrm{cl}(\cdot)$ denotes the closure. $C$ is said to have a bounded (resp., weakly compact) base, if it has a base that is bounded (resp., weakly compact). It is known that $C^{+1} \neq \emptyset$ if and only if $C$ has a base.

Let $A$ be a subset of $A$ and $a$ in $A$. As usual, we denote by $\mathrm{E}(A, C), \mathrm{WE}(A, C)$, and $\operatorname{Pos}(A, C)$ the set of all Pareto points of $A$, the set of all weak Pareto points of $A$, and the set of all positively proper Pareto points of $A$, respectively; that is,

$$
\begin{gathered}
a \in \mathrm{E}(A, C) \Longleftrightarrow(a-C) \cap A=\{a\}, \\
a \in \operatorname{WE}(A, C) \Longleftrightarrow(a-\operatorname{int}(C)) \cap A=\emptyset,
\end{gathered}
$$

$$
\begin{aligned}
a & \in \operatorname{Pos}(A, C) \\
& \Longleftrightarrow \exists c^{*} \in C^{+1} \text { satisfying }\left\langle c^{*}, y-a\right\rangle \geq 0 \quad \forall y \in A .
\end{aligned}
$$

It is clear that

$$
\operatorname{Pos}(A, C) \subset \mathrm{E}(A, C)=\mathrm{E}(A+C, C) \subset \mathrm{WE}(A, C) .
$$

For a multifunction $F: X \rightrightarrows Y$, we denote by $\mathrm{Gr}(F)$ and $\operatorname{epi}_{C}(F)$ the graph and $C$-epigraph of $F$, respectively; that is,

$$
\begin{gathered}
\operatorname{Gr}(F):=\{(x, y): x \in X, y \in F(x)\}, \\
\operatorname{epi}_{C}(F):=\{(x, y): x \in X, y \in F(x)+C\} .
\end{gathered}
$$

We say that $F$ is $C$-convex, if $\operatorname{epi}_{C}(F)$ is convex. Obviously, $F$ is $C$-convex if and only if

$$
\begin{array}{r}
t F\left(x_{1}\right)+(1-t) F\left(x_{2}\right) \subset F\left(t x_{1}+(1-t) x_{2}\right)+C \\
\forall x_{1}, x_{2} \in X, \forall t \in[0,1] .
\end{array}
$$

Recall [14] that a subset $P$ of a Banach space $Z$ is a (convex) polyhedron, if there exist $u_{1}^{*}, \ldots, u_{m}^{*} \in Z^{*}$ and $s_{1}, \ldots, s_{m} \in \mathbb{R}$ such that

$$
P=\left\{z \in Z:\left\langle u_{i}^{*}, z\right\rangle \leq s_{i}, i=1, \ldots, m\right\} .
$$


A subset $Q$ of $Z$ is said to be a G-polyhedron, if $Q$ is a polyhedron, or there exist a polyhedron $P$ of $Z$ and $v_{1}^{*}, \ldots, v_{m}^{*} \in Z^{*}, r_{1}, \ldots, r_{m} \in \mathbb{R}$ such that

$$
Q=P \cap\left\{z \in Z:\left\langle v_{i}^{*}, z\right\rangle<r_{i}, i=1, \ldots, m\right\}
$$

see $[11,13,15]$. Clearly, each polyhedron is closed but a Gpolyhedron is not necessarily closed. From the definitions, it is easy to verify that if $Q_{1}$ and $Q_{2}$ are G-polyhedra of Banach space $Z, Q_{1} \cap Q_{2}$ is a G-polyhedron of $Z$, and $Q_{1} \backslash Q_{2}$ is the union of finitely many G-polyhedra of $Z$.

In this paper, we will consider set-valued vector optimization problem (SVOP). In the remainder of the paper, we always assume that the graph $\operatorname{Gr}(F)$ of the objective mapping $F$ is the union of finitely many G-polyhedra or polyhedra of $X \times Y$.

We say that $\bar{x} \in \Gamma$ is a Pareto (resp., weak Pareto or positively proper Pareto) solution of (SVOP), if there exists $\bar{y} \in F(\bar{x})$ such that $\bar{y} \in \mathrm{E}(F(\Gamma), C)$ (resp., $\bar{y} \in \mathrm{WE}(F(\Gamma), C$ ) or $\bar{y} \in \operatorname{Pos}(F(\Gamma), C))$; in this case, we say that $\bar{y}$ is a Pareto (resp., weak Pareto or positively proper Pareto) optimal value of (SVOP). Let $S, S_{w}$, and $S_{p}$, respectively, denote the sets of all Pareto, weak Pareto and positively proper Pareto solutions of (SVOP). Let $V, V_{w}$, and $V_{p}$, respectively, denote the sets of all Pareto, weak Pareto, and positively proper Pareto optimal values of (SVOP). Obviously,

$$
\begin{gathered}
S_{p} \subset S \subset S_{w}, \quad V_{p} \subset V \subset V_{w}, \\
S_{p}=P \cap F^{-1}\left(V_{p}\right), \quad S=\Gamma \cap F^{-1}(V), \\
S_{w}=\Gamma \cap F^{-1}\left(V_{w}\right) .
\end{gathered}
$$

Next, we provide some properties on the union of finitely many G-polyhedra in general Banach spaces, which generalize the corresponding results in [13].

Lemma 1. A subset $P$ of $X \times Y$ is the union of finitely many G-polyhedra (resp., polyhedra) if and only if there exist closed subspaces $X_{1}$ and $X_{2}$ of $X$, closed subspaces $Y_{1}$ and $Y_{2}$ of $Y$, and the union of finitely many G-polyhedra (resp., polyhedron) $P_{2}$ of $X_{2} \times Y_{2}$ such that

$$
\begin{gathered}
X \times Y=X_{1} \times Y_{1}+X_{2} \times Y_{2}, \\
\left(X_{1} \times Y_{1}\right) \cap\left(X_{2} \times Y_{2}\right)=\{(0,0)\}, \\
\max \left\{\operatorname{dim}\left(X_{2}\right), \operatorname{dim}\left(Y_{2}\right)\right\}<+\infty, \\
P=X_{1} \times Y_{1}+P_{2} .
\end{gathered}
$$

The proof of Lemma 1 is similar to that for polyhedron or G-polyhedron in [13] and is omitted.

If $Y=\{0\}$ in Lemma 1, we have the following corollary.

Corollary 2. A subset $P$ of $X$ is the union of finitely many G-polyhedra (resp., polyhedra) if and only if there exist closed subspaces $X_{1}$ and $X_{2}$ of $X$ and the union of finitely many $G$ polyhedra (resp., polyhedra) $P_{2}$ of $X_{2}$ such that

$$
\begin{gathered}
X=X_{1}+X_{2}, \quad X_{1} \cap X_{2}=\{0\}, \quad \operatorname{dim}\left(X_{2}\right)<+\infty, \\
P=X_{1}+P_{2} .
\end{gathered}
$$

For a subset $A$ of $X \times Y$, let

$$
\begin{aligned}
& A_{X}:=\{x \in X: \exists y \in Y \text { s.t. }(x, y) \in A\}, \\
& A_{Y}:=\{y \in Y: \exists x \in X \text { s.t. }(x, y) \in A\} .
\end{aligned}
$$

Lemma 3 (see [13]). Let $P$ be a G-polyhedron (resp., polyhedron) of $X \times Y$. Then $P_{X}$ is the union of finitely many $G$ polyhedra (resp., polyhedra) of $X$.

Corollary 4. Let $G: X \rightrightarrows Y$ be a multifunction whose graph is a convex G-polyhedron (resp., polyhedra) of $X \times Y$. Let $P$ and $Q$ be convex $G$-polyhedra (resp., polyhedra) of $X$ and $Y$, respectively. Then $G(P)$ and $G^{-1}(Q)$ are the union of finitely many G-polyhedra (resp., polyhedra) of $Y$ and $X$, respectively.

Noting that

$$
\begin{gathered}
G(P)=(\operatorname{Gr}(G) \cap(P \times Y))_{Y}, \\
G^{-1}(Q)=(\operatorname{Gr}(G) \cap(X \times Q))_{X},
\end{gathered}
$$

Corollary 4 is a consequence of Lemma 3.

Lemma 5. Let $A$ be a subset of $Y$ with a closed convex cone $C$. Then

$$
\mathrm{E}(\mathrm{A}, \mathrm{C})=\mathrm{A} \backslash(\mathrm{A}+\mathrm{C} \backslash\{0\})
$$

Proof. Let $a \in A$. Then, one can easily verify that

$$
(a-C \backslash\{0\}) \cap A=\emptyset \Longleftrightarrow a \in A \backslash(A+C \backslash\{0\}) .
$$

Noting that

$$
a \in \mathrm{E}(A, C) \Longleftrightarrow(a-C \backslash\{0\}) \cap A=\emptyset,
$$

it follows that (15) holds. The proof is completed.

Lemma 6. Let $A$ be a subset of Banach space $Y$ with the ordering cone $C$. Then,

$$
W E(A, C)=A \cap W E(\operatorname{cl} A, C) .
$$

Proof. It is obvious that $A \cap \mathrm{WE}(\operatorname{cl} A, C) \subseteq \mathrm{WE}(A, C)$. We only need to show that $\mathrm{WE}(A, C) \subseteq A \cap \mathrm{WE}(\operatorname{cl} A, C)$. Let $x \in \mathrm{WE}(A, C)$. Then $(x-\operatorname{int}(C)) \cap A=\emptyset$; that is

$$
A \subset Y \backslash(x-\operatorname{int}(C)) \text {. }
$$

Since $x-\operatorname{int}(C)$ is open, one has $\operatorname{cl}(A) \subset Y \backslash(x-\operatorname{int}(C))$ and hence,

$$
(x-\operatorname{int}(C)) \cap \operatorname{cl}(A)=\emptyset .
$$

Thus $x \in \mathrm{WE}(\mathrm{cl} A, C)$. The proof is completed. 
We will also use the following lemma [13, Proposition 2.1] in the sequel.

Lemma 7. Let $Y_{1}$ and $Y_{2}$ be closed subspaces of a Banach space $Y$ such that

$$
Y=Y_{1}+Y_{2}, \quad Y_{1} \cap Y_{2}=\{0\} .
$$

Let $A:=Y_{1}+A_{2}$ for some subset $A_{2}$ of $Y_{2}$. Let $C_{2}$ be the projection of the ordering cone $\mathrm{C}$ on $\mathrm{Y}_{2}$; that is,

$$
C_{2}=\left\{c_{2} \in Y_{2}: y_{1}+c_{2} \in C \text { for some } y_{1} \in Y_{1}\right\} \text {. }
$$

Then, the following assertions hold.

(i) $Y_{1}+\operatorname{Pos}\left(A_{2}, C_{2}\right) \subset \operatorname{Pos}(A, C)$ if $Y_{1} \cap C=\{0\}$.

(ii) $\mathrm{E}(A, C)=\emptyset$ if $Y_{1} \cap C \neq\{0\}$.

(iii) $\mathrm{E}(A, C)=Y_{1}+\mathrm{E}\left(A_{2}, C_{2}\right)$ if $Y_{1} \cap C=\{0\}$.

(iv) $W E(A, C)=\emptyset$ if $Y_{1} \cap \operatorname{int}(C) \neq \emptyset$.

(v) $W E(A, C)=Y_{1}+W E\left(A_{2}, C_{2}\right)$ if $\operatorname{int}(C) \neq \emptyset$ and $Y_{1} \cap$ $\operatorname{int}(C)=\emptyset$.

Proof. For the proofs of (ii)-(v), see [13, Proposition 2.1]. We only need to show (i).

For (i), we assume that $Y_{1} \cap C=\{0\}$. Let $y_{1} \in Y_{1}$ and $y_{2} \in \operatorname{Pos}\left(A_{2}, C_{2}\right)$. Then there exists $\widetilde{c}^{*} \in C_{2}^{+1}$ such that

$$
\left\langle\widetilde{c}^{*}, a_{2}-y_{2}\right\rangle \geq 0 \quad \forall a_{2} \in A_{2} .
$$

Define $c^{*}$ by

$$
\left\langle c^{*}, x_{1}+x_{2}\right\rangle:=\left\langle\widetilde{c}^{*}, x_{2}\right\rangle, \quad \forall\left(x_{1}, x_{2}\right) \in Y_{1} \times Y_{2} .
$$

Then $c^{*}$ is well defined and it is easy to verify that $c^{*} \in C^{+1}$. On the other hand, (23) and (24) imply that

$$
\left\langle c^{*}, x_{1}+a_{2}-\left(y_{1}+y_{2}\right)\right\rangle \geq 0 \quad \forall x_{1} \in Y_{1}, \forall a_{2} \in A_{2} \text {. }
$$

Hence $y_{1}+y_{2} \in \operatorname{Pos}(A, C)$.

Remark 8. In view of Lemma 7, it is practical to investigate some topics on (SVOP) in a finite dimensional framework. This is our main ideal to consider (SVOP) in general Banach spaces.

\section{The Structure of Solution Sets and Optimal Value Sets}

In this section, our aim is to investigate the structure of the (weak) Pareto solution set and the (weak) Pareto optimal set of (SVOP) whose graph $\operatorname{Gr}(F)$ is the union of finitely many G-polyhedra. Throughout the remainder of this paper, we assume that $S_{w}, S$, and $S_{p}$ are nonempty.

Let $Y$ be a Banach space. For $y^{*} \in Y^{*}$ and $A \subset Y$, let

$$
\begin{gathered}
\lambda_{y^{*}}(A):=\inf _{a \in A}\left\langle y^{*}, a\right\rangle, \\
L_{y^{*}}(A):=\left\{a \in A:\left\langle y^{*}, a\right\rangle=\inf _{y \in A}\left\langle y^{*}, y\right\rangle\right\} .
\end{gathered}
$$

It is easy to verify that

$$
\operatorname{Pos}(A, C)=\bigcup_{y^{*} \in C^{+1}} L_{y^{*}}(A) .
$$

In addition, if $A+C$ is convex and $C$ has a nonempty interior, by [21, Corollary 5.29], one has

$$
\mathrm{WE}(A, C)=\bigcup_{y^{*} \in C^{+} \backslash\{0\}} L_{y^{*}}(A) .
$$

The following lemma is also useful in our later analysis and can be found in [13].

Lemma 9. Let $Y$ be a Banach space with the ordering cone $C$ which has a weakly compact base. Let $A$ be the union of finitely many polyhedra of $Y$. Suppose that $A+C$ is convex and that $\mathrm{E}(A, C)$ is nonempty. Then $\mathrm{E}(A, C)$ is the union of finitely many convex polyhedra. More precisely, there exist $y_{1}^{*}, \ldots, y_{p}^{*} \in C^{+1}$ such that

$$
\mathrm{E}(\mathrm{A}, \mathrm{C})=\operatorname{Pos}(\mathrm{A}, \mathrm{C})=\bigcup_{\mathrm{i}=1}^{\mathrm{p}} \mathrm{L}_{\mathrm{y}_{\mathrm{i}}^{*}}(\mathrm{~A}) .
$$

We have the following proposition.

Proposition 10. Let $Y$ be a Banach space with the ordering cone $C$ and let $A:=\bigcup_{i=1}^{k} A_{i}, A_{i} \subset Y$. Set

$$
\begin{aligned}
& E_{i} \\
& :=E\left(A_{i}, C\right) \backslash \bigcup_{i \leq j \leq k, j \neq i}\left[\left(A_{j}+C\right) \backslash\left(E\left(A_{i}, C\right) \cap E\left(A_{j}, C\right)\right)\right] .
\end{aligned}
$$

Then, $\mathrm{E}(\mathrm{A}, \mathrm{C})=\bigcup_{\mathrm{i}=1}^{\mathrm{k}} \mathrm{E}_{\mathrm{i}}$.

Proof. Let $x \in \mathrm{E}(A, C)$. Then, there exists an integer $i_{0} \in[1-$ $k$ ] such that $x \in \mathrm{E}\left(A_{i_{0}}, C\right)$. For each $j$ with $x \in A_{j}+C$, noting that

$$
\begin{aligned}
\left(A_{j}\right. & +C) \cap \mathrm{E}(A, C) \\
& =\left(A_{j}+C\right) \cap \mathrm{E}(A+C, C) \subset \mathrm{E}\left(A_{j}+C, C\right) \\
& =\mathrm{E}\left(A_{j}, C\right),
\end{aligned}
$$

one has $x \in \mathrm{E}\left(A_{j}, C\right)$ and $x \in \mathrm{E}\left(A_{i_{0}}, C\right) \cap \mathrm{E}\left(A_{j}, C\right)$. It follows that

$$
x \notin\left(A_{j}+C\right)\left(\mathrm{E}\left(A_{i_{0}}, C\right) \cap \mathrm{E}\left(A_{j}, C\right)\right)
$$$$
\text { for each integer } j \in[1-k] \text {. }
$$

Therefore, one has $x \in E_{i_{0}}$ which implies that $\mathrm{E}(A, C) \subset$ $\bigcup_{i=1}^{k} E_{i}$.

Conversely, let $x \in \bigcup_{i=1}^{k} E_{i}$. Then, $x \in E_{i}$ for some integer $i$ in $[1-k]$. Let $z \in A \cap(x-C)$. We only need to show that $z=x$. By the definition of $A$, there exists an integer $j$ in $[1-k]$ such that $z \in A_{j}$. Noting that $E_{i} \subset \mathrm{E}\left(A_{i}, C\right)$, one has $z=x$ 
if $j=i$. Now suppose that $j \neq i$. Then, by the definition of $E_{i}$, it follows that $x \notin\left(A_{j}+C\right) \backslash\left(\mathrm{E}\left(A_{i}, C\right) \cap \mathrm{E}\left(A_{j}, C\right)\right)$. Since $x \in z+C \subset A_{j}+C$, one has $x \in \mathrm{E}\left(A_{j}, C\right)$. It follows that $x=z$. The proof is completed.

Lemma 11. Let $Y$ be a Banach space, let $A$ be the union of finitely many G-polyhedra of $Y$, and let $\Lambda$ be a subset of $Y^{*}$. Then, $\bigcup_{y^{*} \in \Lambda} L_{y^{*}}(A)$ is the union of finitely many G-polyhedra of $Y$ and, more precisely, there exists a finite subset $\Lambda_{0}$ of $\Lambda$ such that

$$
\bigcup_{y^{*} \in \Lambda} L_{y^{*}}(A)=\bigcup_{y^{*} \in \Lambda_{0}} L_{y^{*}}(A)
$$

Proof. By the assumptions, there exist finitely many Gpolyhedra $P_{1}, \ldots, P_{n}$ such that $A=\bigcup_{i=1}^{n} P_{i}$. Then for each $i \in[1-n]$, let

$$
\Lambda_{i}:=\left\{y^{*} \in \Lambda: P_{i} \cap L_{y^{*}}(A) \neq \emptyset\right\} .
$$

Then

$$
\bigcup_{y^{*} \in \Lambda} L_{y^{*}}(A)=\bigcup_{i=1}^{n}\left(\bigcup_{y^{*} \in \Lambda_{i}} P_{i} \cap L_{y^{*}}(A)\right) .
$$

Let $y^{*} \in \Lambda_{i}$. Since $\lambda_{y^{*}}(A)=\lambda_{y^{*}}(\mathrm{cl}(A))$ and $\mathrm{cl}\left(P_{i}\right)$ is a polyhedron, (26) implies that $\mathrm{cl}\left(P_{i}\right) \cap L_{y^{*}}(A)$ is a face of $\mathrm{cl}\left(P_{i}\right)$. Noting that every polyhedron has finitely many faces. Hence, there exists a finite subset $\widetilde{\Lambda}_{i}$ of $\Lambda_{i}$, such that

$$
\begin{aligned}
\left\{\operatorname{cl}\left(P_{i}\right)\right. & \left.\cap L_{y^{*}}(A): y^{*} \in \Lambda_{i}\right\} \\
= & \left\{\operatorname{cl}\left(P_{i}\right) \cap L_{y^{*}}(A): y^{*} \in \widetilde{\Lambda}_{i}\right\} .
\end{aligned}
$$

Now, we show that for any $y_{1}^{*}, y_{2}^{*} \in \Lambda_{i}$,

$$
\begin{aligned}
& \mathrm{cl}\left(P_{i}\right) \cap L_{y_{1}^{*}}(A)=\operatorname{cl}\left(P_{i}\right) \cap L_{y_{2}^{*}}(A) \\
& \text { implies } P_{i} \cap L_{y_{1}^{*}}(A)=P_{i} \cap L_{y_{2}^{*}}(A) .
\end{aligned}
$$

If (37) holds, by (36), we have

$$
\bigcup_{y^{*} \in \Lambda_{i}} P_{i} \cap L_{y^{*}}(A)=\bigcup_{y^{*} \in \widetilde{\Lambda}_{i}} P_{i} \cap L_{y^{*}}(A) .
$$

From (35), it follows that

$$
\bigcup_{y^{*} \in \Lambda} L_{y^{*}}(A)=\bigcup_{i=1}^{n}\left(\bigcup_{y^{*} \in \widetilde{\Lambda}_{i}} P_{i} \cap L_{y^{*}}(A)\right) .
$$

Let $\Lambda_{0}:=\bigcup_{i=1}^{n} \widetilde{\Lambda}_{i}$. Noting that $P_{i} \cap L_{y^{*}}(A) \subset L_{y^{*}}(A)$, we have that (33) holds. Hence, we only need to show (37). Suppose there would exist $y_{1}^{*}, y_{2}^{*} \in \Lambda_{i}$ such that $\operatorname{cl}\left(P_{i}\right) \cap L_{y_{1}^{*}}(A)=$ $\operatorname{cl}\left(P_{i}\right) \cap L_{y_{2}^{*}}(A)$, but $P_{i} \cap L_{y_{1}^{*}}(A) \neq P_{i} \cap L_{y_{2}^{*}}(A)$. Let $y \in P_{i} \cap$ $L_{y_{1}^{*}}(A)$ with $y \notin P_{i} \cap L_{y_{2}^{*}}(A)$. By (26) and the definition of $\Lambda_{i}$, we have $\left\langle y_{2}^{*}, y\right\rangle>\lambda_{y^{*}}(A)$, which implies that $y \notin \mathrm{cl}\left(P_{i}\right) \cap$ $\lambda_{y_{2}^{*}}(A)=\operatorname{cl}\left(P_{i}\right) \cap \lambda_{y_{1}^{*}}(A)$. This is a contradiction. The proof is completed.
Theorem 12. Let $X$ and $Y$ be Banach spaces, $\Gamma$ a G-polyhedron, and $\operatorname{Gr}(F)$ the union of finitely many $G$-polyhedra of $X \times Y$ and let the ordering cone $C$ have nonempty interior. Suppose that $F(\Gamma)+C$ is convex. Then, $S_{w}$ and $V_{w}$ are the union of finitely many $G$-polyhedra of $X$ and $Y$, respectively. More precisely, there exist $y_{i}^{*} \in C^{+}$with $\left\|y_{i}^{*}\right\|=1(i \in[i-q]$ for some integer q) such that

$$
V_{w}=\bigcup_{y^{*} \in C^{+} \backslash\{0\}} L_{y^{*}}(F(\Gamma))=\bigcup_{i=1}^{q} L_{y_{i}^{*}}(F(\Gamma)) .
$$

Proof. Since $F(\Gamma)+C$ is convex, by (28), we have

$$
\mathrm{WE}(F(\Gamma)+C, C)=\bigcup_{y^{*} \in C^{+} \backslash\{0\}} L_{y^{*}}(F(\Gamma)+C) .
$$

Noting that $\operatorname{WE}(F(\Gamma), C)=F(\Gamma) \cap \operatorname{WE}(F(\Gamma)+C, C)$, we have

$$
\begin{aligned}
\operatorname{WE}( & F(\Gamma), C) \\
& =\bigcup_{y^{*} \in C^{+} \backslash\{0\}} F(\Gamma) \cap L_{y^{*}}(F(\Gamma)+C) \\
& =\bigcup_{y^{*} \in C^{+} \backslash\{0\}} L_{y^{*}}(F(\Gamma)) .
\end{aligned}
$$

Since the feasible set $\Gamma$ is a G-polyhedron of $X$ and $\operatorname{Gr}(F)$ is the union of finitely many G-polyhedra of $X \times Y$, Corollary 4 implies that $F(\Gamma)$ is the union of finitely many G-polyhedra of $Y$. Since $V_{w}=\mathrm{WE}(F(\Gamma), C)$, it follows from (26) and Lemma 11 that $V_{w}$ is the union of finitely many G-polyhedra of $Y$ and there exist $y_{i}^{*} \in C^{+}$with $\left\|y_{i}^{*}\right\|=1(i \in[i-q]$ for some integer $q$ ) such that (40) holds. On the other hand, from Corollary 4 , one can easily see that $S_{w}=\Gamma \cap F^{-1}\left(V_{w}\right)$ is the union of finitely many G-polyhedra of $X$. The proof is completed.

By Lemma 3, dropping convexity of $F(\Gamma)+C$ but requiring that the ordering cone $C$ is polyhedral, we generalize Theorem 3.3 in [13] to the union of finitely many G-polyhedra setting.

Theorem 13. Let $X$ and $Y$ be Banach spaces. Let the ordering cone $C$ be polyhedral and have nonempty interior. Suppose that $\Gamma$ and $\operatorname{Gr}(F)$ are the unions of finitely many G-polyhedra of $X$ and $X \times Y$, respectively. Then, $S_{w}$ and $V_{w}$ are the unions of finitely many $G$-polyhedra of $X$ and $Y$, respectively.

Proof. Suppose that $\operatorname{Gr}(F)$ is the union of finitely many Gpolyhedra of $X \times Y$. Since $F(\Gamma)=(\operatorname{Gr}(F) \cap(\Gamma \times Y))_{Y}$, by Lemma 3, one has that $\operatorname{cl}(F(\Gamma))$ is the union of finitely many polyhedra of $Y$. It is similar to the proof of Theorem 3.3 in [13]; we can show that $\mathrm{WE}(\operatorname{cl}(F(\Gamma)))$ is the union of finitely many polyhedra of $Y$. By Lemma 6 , one has

$$
V_{w}=\mathrm{WE}(F(\Gamma), C)=F(\Gamma) \cap \operatorname{WE}(\operatorname{cl}(F(\Gamma)), C)
$$

which implies that $V_{w}$ is the union of finitely many Gpolyhedra of $Y$. Noting that $S_{w}=\left(\operatorname{Gr}(F) \cap(\Gamma) \times V_{w}\right)_{X}$, Lemma 3 implies that $S_{w}$ is the union of finitely many Gpolyhedra of $X$. The proof is completed. 
Dropping the assumption of the ordering cone $C$ having a weakly compact base but inquiring $C$ being a polyhedral cone, we have the following result analogous to that of Lemma 11.

Lemma 14. Let $Y$ be a Banach space, let the ordering cone $C$ be pointed and polyhedral, and let $A$ be the union of finitely many polyhedra of $Y$. Suppose that $A+C$ is convex and that $\mathrm{E}(A, C)$ is nonempty. Then $\mathrm{E}(A, C)$ is the union of finitely many polyhedra of $Y$. More precisely, there exist $c_{1}^{*}, \ldots, c_{p}^{*} \in$ $C^{+1}$ such that

$$
\begin{gathered}
E(A, C)=\operatorname{Pos}(A, C)=\bigcup_{i=1}^{p} L_{c_{i}^{*}}(A), \\
L_{c_{i}^{*}}(\mathrm{cl} \cos A)=L_{c_{i}^{*}}(A) .
\end{gathered}
$$

Proof. By Corollary 2 there exist a closed subspace $Y_{1}$, a finite dimensional subspace $Y_{2}$ of $Y$, and the union $A_{2}$ of finitely many polyhedra of $Y_{2}$ such that

$$
\begin{gathered}
Y=Y_{1}+Y_{2}, \quad Y_{1} \cap Y_{2}=\{0\}, \\
A=Y_{1}+A_{2} .
\end{gathered}
$$

Let $C_{2}$ be the projection of the ordering cone $C$ on $Y_{2}$; that is,

$$
C_{2}=\left\{c_{2} \in Y_{2}: c_{1}+c_{2} \in C \text { for some } c_{1} \in Y_{1}\right\} \text {. }
$$

Since $C$ is polyhedral, together with Corollary 4 , we have that the cone $C_{2}$ is the union of finitely many polyhedra of $Y_{2}$. Hence it is also closed. From the assumption of $\mathrm{E}(A, C) \neq \emptyset$, by Lemma 7(ii), we have $Y_{1} \cap C=\{0\}$. From this, with Lemma 7 (iii), we have $\mathrm{E}\left(A_{2}, C_{2}\right) \neq \emptyset$. By Lemma 7(i), we have

$$
\operatorname{Pos}(A, C) \supset Y_{1}+\operatorname{Pos}\left(A_{2}, C_{2}\right) \text {. }
$$

We claim that $\operatorname{Pos}\left(A_{2}, C_{2}\right)$ is the union of finitely many polyhedra of $Y_{2}$. For any $(x, c) \in A \times C$, there exist $\left(x_{1}, x_{2}\right) \in$ $Y_{1} \times A_{2}$ and $\left(c_{1}, c_{2}\right) \in Y_{1} \times C_{2}$ such that

$$
x=x_{1}+x_{2}, \quad c=c_{1}+c_{2} .
$$

Since $Y_{1}$ is a subspace of $Y$, we have

$$
x+c=\left(x_{1}+c_{1}\right)+x_{2}+c_{2} \in Y_{1}+A_{2}+C_{2} .
$$

Hence we have

$$
A+C \subset Y_{1}+A_{2}+C_{2} \text {. }
$$

Conversely, for any $\left(x_{1}, a_{2}, c_{2}\right) \in Y_{1} \times A_{2} \times C_{2}$, there exists $c_{1} \in Y_{1}$ such that $c_{1}+c_{2} \in C$. Hence we have

$$
\begin{aligned}
y_{1}+a_{2} & +c_{2} \\
& =\left(y_{1}-c_{1}\right)+a_{2}+\left(c_{1}+c_{2}\right) \in Y_{1}+A_{2}+C \\
& =A+C .
\end{aligned}
$$

Thus we have shown that

$$
A+C=Y_{1}+A_{2}+C_{2} .
$$

Let $x_{2}, y_{2} \in A_{2}+C_{2}$. Then there exist $x_{1}, y_{1} \in Y_{1}$ such that

$$
x_{1}+x_{2}, y_{1}+y_{2} \in A+C
$$

Since $A+C$ is convex, by (53), one has

$$
\begin{array}{r}
\lambda\left(x_{1}+x_{2}\right)+(1-\lambda)\left(y_{1}+y_{2}\right) \in Y_{1}+\left(A_{2}+C_{2}\right) \\
\forall \lambda \in[0,1] .
\end{array}
$$

Noting that

$$
\begin{gathered}
\lambda x_{1}+(1-\lambda) y_{1} \in Y_{1}, \\
\lambda x_{2}+(1-\lambda) y_{2} \in Y_{2} \quad \forall \lambda \in[0,1],
\end{gathered}
$$

from (45) and (55), one has

$$
\lambda x_{2}+(1-\lambda) y_{2} \in A_{2}+C_{2} \quad \forall \lambda \in[0,1] .
$$

Hence $A_{2}+C_{2}$ is convex.

Let $x_{2} \in C_{2} \cap-C_{2}$. Then there exist $x_{1}^{\prime}, x_{1}^{\prime \prime} \in Y_{1}$ such that $x_{1}^{\prime}+x_{2}, x_{1}^{\prime \prime}-x_{2} \in C$. Since $C$ is a convex cone, it follows that

$$
x_{1}^{\prime}+x_{1}^{\prime \prime}=\left(x_{1}^{\prime}+x_{2}\right)+\left(x_{1}^{\prime \prime}-x_{2}\right) \in C .
$$

Hence

$$
x_{1}^{\prime}+x_{1}^{\prime \prime} \in Y_{1} \cap C=\{0\} .
$$

We have

$$
x_{1}^{\prime \prime}=-x_{1}^{\prime}, \quad x_{1}^{\prime}+x_{2} \in C \cap-C=\{0\} .
$$

Together with (46), we have $x_{2}=0$ and $C_{2} \cap-C_{2}=\{0\}$.

We have shown that $C_{2}$ is a closed, convex, and pointed cone of $Y_{2}$. Noting that $Y_{2}$ is finite dimensional, it follows that $C_{2}$ has a compact base. Applying Lemma 9, it follows that there exist $\widetilde{c}_{1}^{*}, \ldots, \widetilde{c}_{p}^{*}$ in $C_{2}^{+1}$ such that

$$
\operatorname{Pos}\left(A_{2}, C_{2}\right)=\mathrm{E}\left(A_{2}, C_{2}\right)=\bigcup_{i=1}^{p} L_{\widetilde{c}_{i}^{*}}\left(A_{2}\right) .
$$

Thus $\operatorname{Pos}\left(A_{2}, C_{2}\right)$ is the union of finitely many polyhedra of $Y_{2}$. By Lemma 7((ii), (iii)), (48), and (61), one has that

$$
\begin{aligned}
& \operatorname{Pos}(A, C) \supset Y_{1}+\operatorname{Pos}\left(A_{2}, C_{2}\right) \\
& =Y_{1}+\mathrm{E}\left(A_{2}, C_{2}\right)=\mathrm{E}(A, C) .
\end{aligned}
$$

Hence,

$$
\mathrm{E}(A, C)=\operatorname{Pos}(A, C)=Y_{1}+\operatorname{Pos}\left(A_{2}, C_{2}\right) .
$$

From (45), (46), and (63), applying Corollary 2, it follows that $\operatorname{Pos}(A, C)$ is the union of finitely many polyhedra of $Y$.

For each $\widetilde{c}_{i}^{*}, i \in[1-p]$, we define $c^{*}$ by

$$
\left\langle c_{i}^{*}, y_{1}+y_{2}\right\rangle:=\left\langle\widetilde{c}_{i}^{*}, y_{2}\right\rangle, \quad \forall\left(y_{1}, y_{2}\right) \in Y_{1} \times Y_{2} .
$$

Then, one can easily show that $c_{i}^{*} \in C^{+1}$ and

$$
L_{c_{i}^{*}}(A)=Y_{1}+L_{\widetilde{c}_{i}^{*}}\left(A_{2}\right) \text {. }
$$


In fact, for any $y \in C \backslash\{0\}$, there exists a pair $\left(y_{1}, y_{2}\right) \in Y_{1} \times C_{2}$ such that $y=y_{1}+y_{2}$. If $y_{2}=0$, then $y=y_{1} \in Y_{1}$ and thus $y \in Y_{1} \cap C=\{0\}$, a contradiction. Hence $y_{2} \neq 0$. Then we have

$$
\left\langle c_{i}^{*}, y\right\rangle=\left\langle c_{i}^{*}, y_{1}+y_{2}\right\rangle=\left\langle\widetilde{c}_{i}^{*}, y_{2}\right\rangle>0 .
$$

It follows that $c_{i}^{*} \in C^{+1}$. For (65), let $a \in L_{c_{i}^{*}}(A)$. By (26), (46), and (64), there exists $\left(a_{1}, a_{2}\right) \in Y_{1} \times A_{2}$ such that $a=a_{1}+a_{2}$ and

$$
\begin{aligned}
\left\langle\widetilde{c}_{i}^{*}, a_{2}\right\rangle & =\left\langle c_{i}^{*}, a_{1}+a_{2}\right\rangle \\
& =\inf _{y_{1} \in Y_{1}, y_{2} \in A_{2}}\left\langle c_{i}^{*}, y_{1}+y_{2}\right\rangle=\inf _{y_{2} \in A_{2}}\left\langle\widetilde{c}_{i}^{*}, y_{2}\right\rangle .
\end{aligned}
$$

We have $a_{2} \in L_{\widetilde{c}_{i}^{*}}\left(A_{2}\right)$ and $a \in Y_{1}+L_{\widetilde{c}_{i}^{*}}\left(A_{2}\right)$. The conclusion, $Y_{1}+L_{\widetilde{c}_{i}^{*}}\left(A_{2}\right) \subset A$ holds obviously. Thus (65) holds. From (48) and (63)-(65), we have

$$
\begin{aligned}
\mathrm{E}(A, C) & =\operatorname{Pos}(A, C)=Y_{1}+\bigcup_{i=1}^{p} L_{\widetilde{c}_{i}^{*}}\left(A_{2}\right) \\
& =\bigcup_{i=1}^{p}\left(Y_{1}+L_{\widetilde{c}_{i}^{*}}\left(A_{2}\right)\right)=\bigcup_{i=1}^{p} L_{c_{i}^{*}}(A) .
\end{aligned}
$$

Finally, we show that $L_{c_{i}^{*}}(\mathrm{cl} \operatorname{co} A)=L_{c_{i}^{*}}(A)$. Indeed, for any $y \in Y$, (45) implies that there exists unique pair $\left(y_{1}, y_{2}\right) \in$ $Y_{1} \times Y_{1}$, such that $y=y_{1}+y_{2}$. Since $Y_{2}$ is finitely dimensional, one can take a constant $M>0$ which is independent on $y$ satisfying

$$
\left\|y_{1}\right\|+\left\|y_{2}\right\| \leq M\|y\|
$$

From (46), we have

$$
\operatorname{co} A=Y_{1}+\operatorname{co} A_{2} \subset Y_{1}+\operatorname{cl} \operatorname{co} A_{2} .
$$

It follows that

$$
y_{1}+\mathrm{cl} \operatorname{co} A_{2} \subset \mathrm{cl} \operatorname{co} A \quad \forall y_{1} \in Y_{1},
$$

and thus

$$
Y_{1}+\mathrm{cl} \operatorname{co} A_{2} \subset \mathrm{cl} \operatorname{co} A .
$$

On the other hand, for each sequenc $\left\{\left(y_{1, n}, a_{2, n}\right)\right\} \subset Y_{1} \times$ cl co $A_{2}$ with $y_{n}:=y_{1, n}+a_{2, n}$ converging to some $y \in Y$, there exists a pair $\left(y_{1}, y_{2}\right) \in Y_{1} \times Y_{2}$ such that $y=y_{1}+y_{2}$. Together with (69), we have

$$
\left\|y_{1, n}-y_{1}\right\|+\left\|y_{2, n}-y_{2}\right\| \leq M\left\|y_{n}-y\right\|,
$$

and hence $\left\{y_{1, n}\right\}$ and $\left\{y_{2, n}\right\}$ converge to $y_{1} \in Y_{1}$ (since $Y_{1}$ is closed) and $y_{2} \in \mathrm{cl} \operatorname{co} A_{2}$, respectively. Thus we have $y \in$ $Y_{1}+\mathrm{cl} \operatorname{co} A_{2}$ and hence $Y_{1}+\mathrm{cl} \operatorname{co} A_{2}$ is closed. Together with (70), we have

$$
\mathrm{cl} \operatorname{co} A \subset Y_{1}+\mathrm{cl} \operatorname{co} A_{2} .
$$

From (72) and (74), we have

$$
\text { cl co } A=Y_{1}+\mathrm{cl} \operatorname{co} A_{2},
$$

and together with (65), one has

$$
L_{c_{i}^{*}}(\mathrm{cl} \operatorname{co} A)=Y_{1}+L_{\widetilde{c}_{i}^{*}}\left(\operatorname{cl} \operatorname{co} A_{2}\right) .
$$

By Theorem 3.2(i) in [13], it follows that

$$
L_{\widetilde{c}_{i}^{*}}\left(\operatorname{cl} \operatorname{co} A_{2}\right)=L_{\widetilde{c}_{i}^{*}}\left(A_{2}\right) .
$$

This and (65) imply that

$$
\begin{aligned}
L_{c_{i}^{*}}(\operatorname{clco} A) & =Y_{1}+L_{\widetilde{c}_{i}^{*}}\left(\operatorname{clco} A_{2}\right) \\
& =Y_{1}+L_{\widetilde{c}_{i}^{*}}\left(A_{2}\right)=L_{c_{i}^{*}}(A) .
\end{aligned}
$$

The proof is completed.

Theorem 15. Let $X$ and $Y$ be Banach spaces and let the ordering cone $C$ be pointed and polyhedral. Suppose that $\Gamma$ and $\mathrm{Gr}(\mathrm{F})$ are the unions of finitely many polyhedra of $X$ and $X \times Y$, respectively, and that $F(\Gamma)+C$ is convex. Then, $S$ and $V$ are the union of finitely many convex polyhedra of $X$ and $Y$, respectively, and more precisely, there exist $c_{i}^{*} \in C^{+1}(i \in[1-q]$ for some integer q) such that

$$
V=V_{p}=\bigcup_{i=1}^{q} L_{c_{i}^{*}}(F(\Gamma)) .
$$

Consequently, $S=S_{p}$ and $V$ are the unions of finitely many polyhedra.

Proof. Suppose that $\Gamma$ and $\operatorname{Gr}(F)$ are the unions of finitely many polyhedra of $X$ and $X \times Y$, respectively. Noting that

$$
F(\Gamma)=(\operatorname{Gr}(F) \cap(\Gamma \times Y))_{Y},
$$

and by Lemma 3, one has that $F(\Gamma)$ is the union of finitely many polyhedra of $Y$. From this and Lemma 14, it follows that there exist $c_{i}^{*} \in C^{+1}(i \in[1-q]$ for some integer $q)$ such that (79) holds. Hence, $V=V_{p}$ is the union of finitely many polyhedra of $Y$. This and Corollary 4 imply that $S=S_{p}=$ $\Gamma \cap F^{-1}(V)$ is the union of finitely many polyhedra of $X$. The proof is completed.

Without the convexity of $A+C$ in Lemma 14, we have the following lemma which will also be applied to consider (SVOP).

Lemma 16. Let $Y$ be a Banach space, let the ordering cone $C$ be pointed and polyhedral, and let $A$ be the union of finitely many $G$-polyhedra of $Y$. Suppose that $\mathrm{E}(A, C)$ is nonempty. Then $\mathrm{E}(A, C)$ is the union of finitely many $G$-polyhedra of $Y$.

Proof. By Corollary 2 there exist a closed subspace $Y_{1}$, a finite dimensional subspace $Y_{2}$ of $Y$, and the union $A_{2}$ of finitely many G-polyhedra of $Y_{2}$, such that (45) and (46) hold.

Let $C$ be the projection of $C$ on $Y_{2}$. We first show that $A_{2}+$ $\mathrm{C}_{2}$ is the union of finitely many G-polyhedra of $Y_{2}$. Indeed, since $C$ is a polyhedron on $Y_{1} \times Y_{2}$, Lemma 3 implies that $C_{2}$ is the union of finitely many polyhedra of $Y_{2}$. We can assume

$$
A_{2}=\bigcup_{i=1}^{p} A_{2, i}, \quad C_{2}=\bigcup_{j=1}^{q} C_{2, j},
$$


where each $A_{2, i}$ is a G-polyhedron of $Y_{2}$ and each $C_{2, j}$ is a polyhedron of $Y_{2}$. It follows that

$$
A_{2}+C_{2}=\bigcup_{i=1}^{p} \bigcup_{j=1}^{q}\left(A_{2, i}+C_{2, j}\right) .
$$

Noting that $Y_{2}$ is finitely dimensional, by Proposition 2.1 in [15], we have that each $A_{2, i}+C_{2, j}$ is a G-polyhedron of $Y_{2}$. This with (82) implies that $A_{2}+C_{2}$ is the union of finitely many G-polyhedra of $Y_{2}$. Together with (45), (46), and (53), applying Corollary 2, it follows that $A+C$ is the union of finitely many G-polyhedra of $Y$. Let $A=\bigcup_{i=1}^{k} A_{i}$, where each $A_{i}$ is a G-polyhedron of $Y$. Noting that $\{0\}$ is a face of $C$, by the above proof, applying Proposition 2.1 in [15] again, we have that each $A_{i}+C \backslash\{0\}$ as well as $A_{i}+C$ is the union of finitely many G-polyhedra of $Y$. So is the complimentary $\left(A_{i}+C \backslash\{0\}\right)^{c}$. By Lemma 5, we have

$$
\begin{aligned}
\left(A_{i}, C\right) & =A_{i} \backslash\left(A_{i}+C \backslash\{0\}\right) \\
& =A_{i} \cap\left(A_{i}+C \backslash\{0\}\right)^{c},
\end{aligned}
$$

which implies that $\left(A_{i}, C\right)$ is the union of finitely many Gpolyhedra of $Y$. So is the set

$$
\bigcup_{i \leq j \leq k, j \neq i}\left[\left(A_{j}+C\right) \backslash\left(\mathrm{E}\left(A_{i}, C\right) \cap \mathrm{E}\left(A_{j}, C\right)\right)\right] .
$$

Let

$$
\begin{aligned}
& E_{i} \\
& :=\mathrm{E}\left(A_{i}, C\right) \backslash \bigcup_{i \leq j \leq k, j \neq i}\left[\left(A_{j}+C\right) \backslash\left(\mathrm{E}\left(A_{i}, C\right) \cap \mathrm{E}\left(A_{j}, C\right)\right)\right] .
\end{aligned}
$$

Then $E_{i}$ is also the union of finitely many G-polyhedra of $Y$. Applying Proposition 10, we have

$$
\mathrm{E}(A, C)=\bigcup_{i=1}^{k} E_{i}
$$

Thus $\mathrm{E}(A, C)$ is the union of finitely many $\mathrm{G}$-polyhedra of $Y$. The proof is completed.

Theorem 17. Let $X$ and $Y$ be Banach spaces and let the ordering cone $C$ be pointed and polyhedral. Suppose that $\Gamma$ and $\operatorname{Gr}(F)$ are the unions of finitely many $G$-polyhedra of $X$ and $X \times Y$, respectively. Then, $S$ and $V$ are the union of finitely many convex $G$-polyhedra of $X$ and $Y$, respectively.

Proof. Suppose that $\Gamma$ and $\operatorname{Gr}(F)$ are the unions of finitely many G-polyhedra of $X$ and $X \times Y$, respectively. Noting that

$$
F(\Gamma)=(\operatorname{Gr}(F) \cap(\Gamma \times Y))_{Y}
$$

and by Lemma 3, one has that $F(\Gamma)$ is the union of finitely many polyhedra of $Y$. From this and Lemma 16, we have that $V$ is the union of finitely many G-polyhedra of $Y$. This and Corollary 4 imply that $S=\Gamma \cap F^{-1}(V)$ is the union of finitely many G-polyhedra of $X$ too. The proof is completed.

\section{Connectedness of Pareto Solution Sets and Pareto Optimal Value Sets}

For various applications, it is of special interest to move continuously from an optimal solution to another along optimal alternatives. In order to do this movement, one needs that the optimal solution set is pathwise connected or at least connected. Many authors studied connectedness of optimal solution sets in multiobjective optimization under some restrictive assumptions (cf. [2, 16-20, 22, 23]).

In this section, dropping the assumption that the ordering cone $C$ has a weakly compact base but requiring that $C$ is pointed and polyhedral, under the $C$-convexity assumption on the set-valued objective mapping $F$, we will establish connectedness of Pareto solution set $S$ and Pareto optimal value set $V$ of (SVOP).

Let $F^{\prime}: X \rightrightarrows Y$ be such that

$$
\operatorname{Gr}\left(F^{\prime}\right)=\operatorname{cl} \operatorname{co}(\operatorname{Gr}(F) \cap(\Gamma \times Y))
$$

Consider the following vector optimization problem:

$$
C-\min F^{\prime}(x) \quad \text { subject to } x \in \Gamma \text {. }
$$

Let $S^{\prime}$ and $V^{\prime}$ denote the set of all Pareto solutions of $\left(\right.$ SVOP $\left.^{\prime}\right)$ and the set of all Pareto optimal values of $\left(\mathrm{SVOP}^{\prime}\right)$, respectively.

Lemma 18. Let $\Gamma$ be a polyhedron of $X$, let $C$ be a closed, convex, pointed, and polyhedral cone of $Y$, and let $\operatorname{Gr} F(\Gamma)$ be the union of finitely many polyhedra of $X \times Y$. Suppose that $F$ is $C$-convex. Then $S^{\prime}=S$ and $V^{\prime}=V$.

Proof. By Corollary 4, $F(\Gamma)$ is the union of finitely many polyhedra of $Y$. From Corollary 2, there exist a closed subspace $Y_{1}$, a finite dimensional subspace of $Y$, and the union of finitely many polyhedra $F(\Gamma)_{2}$ of $Y_{2}$ such that

$$
\begin{gathered}
Y=Y_{1}+Y_{2}, \quad Y_{1} \cap Y_{2}=\{0\}, \\
F(\Gamma)=Y_{1}+F(\Gamma)_{2} .
\end{gathered}
$$

By (75) in the proof of Lemma 14, we have

$$
\mathrm{cl} \operatorname{co} F(\Gamma)=Y_{1}+\mathrm{cl} \operatorname{co} F(\Gamma)_{2}
$$

Since $\Gamma$ is a polyhedron of $X$, by Lemma 2.1 in [13] and Theorem 19.6 in [14], $\operatorname{Gr}\left(F^{\prime}\right)$ is a polyhedron of $X \times Y$. By Lemma $3, F^{\prime}(\Gamma)$ is the union of finitely many polyhedra of $X$. Hence it is closed. On the other hand, From the convexity of $\operatorname{Gr}\left(F^{\prime}\right)$, we have that $F^{\prime}(\Gamma)$ is convex. Noting that $F(\Gamma) \subset$ $F^{\prime}(\Gamma) \subset \mathrm{cl} \operatorname{co} F(\Gamma)$, it follows that $F^{\prime}(\Gamma)=\mathrm{cl} \operatorname{co} F(\Gamma)$. Thus by (90), one has

$$
F^{\prime}(\Gamma)=Y_{1}+\mathrm{cl} \operatorname{co} F(\Gamma)_{2}
$$


Let $C_{2}$ be the projection of $C$ on $Y_{2}$. Analogously to the proof of Lemma 14, one can show

$$
\begin{aligned}
V^{\prime} & =\mathrm{E}\left(F^{\prime}(\Gamma), C\right)=\operatorname{Pos}\left(F^{\prime}(\Gamma), C\right) \\
& =Y_{1}+\operatorname{Pos}\left(\mathrm{cl} \operatorname{co} F(\Gamma)_{2}, C_{2}\right), \\
V & =\mathrm{E}(F(\Gamma), C)=\operatorname{Pos}(F(\Gamma), C) \\
& =Y_{1}+\operatorname{Pos}\left(F(\Gamma)_{2}, C_{2}\right) .
\end{aligned}
$$

In order to show $V^{\prime}=V$, we only need to show

$$
\operatorname{Pos}\left(\operatorname{cl} \operatorname{co} F(\Gamma)_{2}, C_{2}\right)=\operatorname{Pos}\left(F(\Gamma)_{2}, C_{2}\right) .
$$

Indeed, since $F$ is $C$-convex, it follows that $F^{\prime}(\Gamma)+C$ and $F(\Gamma)+C$ are convex. It is the same to prove that $A_{2}+C_{2}$ is convex in the proof of Lemma 14; we have that both $F^{\prime}(\Gamma)_{2}+$ $C_{2}$ and $F(\Gamma)_{2}+C_{2}$ are also convex. Noting that $Y_{2}$ is finite dimensional, by Theorem 3.2(i) in [13], one has

$$
L_{\widetilde{c}^{*}}(\mathrm{cl} \operatorname{co} F(\Gamma))=L_{\widetilde{c}^{*}}(F(\Gamma)) \quad \forall \widetilde{c}^{*} \in C_{2}^{+1} .
$$

On the other hand, (27) implies that

$$
\begin{gathered}
\operatorname{Pos}\left(\operatorname{cl} \operatorname{co} F(\Gamma)_{2}, C_{2}\right)=\bigcup_{\tilde{c}^{*} \in C_{2}^{+1}} L_{\widetilde{c}^{*}}\left(\operatorname{cl} \operatorname{co} F(\Gamma)_{2}\right), \\
\operatorname{Pos}\left(F(\Gamma)_{2}, C_{2}\right)=\bigcup_{\widetilde{c}^{*} \in C_{2}^{+1}} L_{\widetilde{c}^{*}}\left(F(\Gamma)_{2}\right) .
\end{gathered}
$$

From (94)-(96), it follows that (93) holds. Hence, we have shown $V^{\prime}=V$. It remains to show $S^{\prime}=S$. Noting that $S^{\prime}=\Gamma \cap F^{\prime-1}\left(V^{\prime}\right), S=\Gamma \cap F^{-1}(V)$, the following assertion in $[13$, see the proof of Lemma 4.1]

$$
\begin{aligned}
\Gamma \cap F^{-1}\left(L_{c^{*}}(F(\Gamma))\right) & =\Gamma \cap F^{\prime-1}\left(L_{c^{*}}\left(F^{\prime}(\Gamma)\right)\right) \quad \forall c^{*} \in C^{+}, \\
V^{\prime} & =\bigcup_{c^{*} \in C^{+1}} L_{c^{*}}\left(F^{\prime}(\Gamma)\right), \\
V & =\bigcup_{c^{*} \in C^{+1}} L_{c^{*}}(F(\Gamma)),
\end{aligned}
$$

it follows that $S^{\prime}=S$. The proof is completed.

For our main result, we need the following lemma; see [13, Lemma 4.2].

Lemma 19. Let $Y$ be a Banach space and A a polyhedron of $Y$. Let $\Lambda$ be a convex subset of $Y^{*}$. Then $\bigcup_{y^{*} \in \Lambda} L_{y^{*}}(A)$ is pathwise connected.

Theorem 20. Let $X$ and $Y$ be Banach spaces, $\Gamma$ a polyhedron of $X, C$ a closed, convex, pointed, and polyhedral cone of $Y$, and $\operatorname{Gr} F(\Gamma)$ the union of finitely many polyhedra of $X \times Y$. Suppose that the set-valued objective function $F$ is $C$-convex. Then, the Pareto solution set $S$ and the Pareto optimal value set $V$ of (SVOP) are pathwise connected.
Proof. Let $F^{\prime}: X \quad X \quad Y$ be defined by (88). Then, $\operatorname{Gr}\left(F^{\prime}\right)$ is a polyhedron of $X \times Y$. By (27), one has $V^{\prime}=$ $\bigcap_{y^{*} \in C^{+1}} L_{y^{*}}\left(F^{\prime}(\Gamma)\right)$. It follows that

$$
\begin{aligned}
\operatorname{Gr}\left(F^{\prime}\right) & \cap\left(\Gamma \times V^{\prime}\right) \\
= & \bigcup_{y^{*} \in C^{+1}} L_{\left(0, y^{*}\right)}\left(\operatorname{Gr}\left(F^{\prime}\right) \cap(\Gamma \times Y)\right) .
\end{aligned}
$$

This and Lemma 19 imply that $\operatorname{Gr}\left(F^{\prime}\right) \cap\left(\Gamma \times V^{\prime}\right)$ is pathwise connected. Hence $S^{\prime}=\left(\operatorname{Gr}\left(F^{\prime}\right) \cap\left(\Gamma \times V^{\prime}\right)\right)_{X}$ and $V^{\prime}=\left(\operatorname{Gr}\left(F^{\prime}\right) \cap\right.$ $\left.\left(\Gamma \times V^{\prime}\right)\right)_{Y}$ are pathwise connected. By Lemma 18, one sees that $S$ and $V$ are pathwise connected.

Corollary 21. Let $Y$ be a Banach space with the pointed ordering $C$ being closed and polyhedral and let $A$ be the union of finitely many convex polyhedra of $Y$. Suppose that $A+C$ is convex. Then, $\mathrm{E}(A, C)$ is pathwise connected.

Remark 22. In [13, Theorem 4.1 and Corollary 4.1], under the assumptions that the ordering cone $C$ has a weakly compact base and the Banach space $Y$ is finite dimensional, respectively, the corresponding results of connectedness of (SVOP) were established. In our results, Theorem 20 and Corollary 21, we drop these two assumptions, respectively.

\section{Acknowledgments}

This research was supported by the National Natural Science Foundation of China (Grant nos. 11261067, 11061039, XT412003) and IRTSTYN.

\section{References}

[1] P. Armand, "Finding all maximal efficient faces in multiobjective linear programming," Mathematical Programming, vol. 61, no. 3, pp. 357-375, 1993.

[2] K. J. Arrow, E. W. Barankin, and D. Blackwell, "Admissible points of convex sets," in Contributions to the Theory of Games, Annals of Mathematics Studies, no. 28, pp. 87-92, Princeton University Press, Princeton, NJ, USA, 1953.

[3] H. P. Benson and E. Sun, "Outcome space partition of the weight set in multiobjective linear programming," Journal of Optimization Theory and Applications, vol. 105, no. 1, pp. 17-36, 2000.

[4] S. I. Gass and P. G. Roy, "The compromise hypersphere for multiobjective linear programming," European Journal of Operational Research, vol. 144, no. 3, pp. 459-479, 2003.

[5] H. W. Hamacher and S. Nickel, "Multiobjecture planar location problems," European Journal of Operational Research, vol. 94, pp. 66-86, 1996.

[6] D. T. Luc, Theory of Vector Optimization, vol. 319 of Lecture Notes in Economics and Mathematical Systems, Springer, Berlin, Germany, 1989.

[7] B. Pérez Gladish, M. Arenas Parra, A. Bilbao Terol, and M. V. Rodríguez Uría, "Management of surgical waiting lists through a possibilistic linear multiobjective programming problem," Applied Mathematics and Computation, vol. 167, no. 1, pp. 477495, 2005. 
[8] L. V. Thuan and D. T. Luc, "On sensitivity in linear multiobjective programming," Journal of Optimization Theory and Applications, vol. 107, no. 3, pp. 615-626, 2000.

[9] M. Zeleny, Linear Multiobjective Programming, vol. 95 of Lecture Notes in Economics and Mathematical Systems, Springer, Berlin, Germany, 1974.

[10] S. Nickel and M. M. Wiecek, "Multiple objective programming with piecewise linear functions," Journal of Multi-Criteria Decision Analysis, vol. 8, pp. 322-332, 1999.

[11] X. Zheng and X. Yang, "The structure of weak Pareto solution sets in piecewise linear multiobjective optimization in normed spaces," Science in China A, vol. 51, no. 7, pp. 1243-1256, 2008.

[12] A. D. Alexandrov, Convex Polyhedra, Springer Monographs in Mathematics, Springer, Berlin, Germany, 2005.

[13] X. Y. Zheng, "Pareto solutions of polyhedral-valued vector optimization problems in Banach spaces," Set-Valued and Variational Analysis, vol. 17, no. 4, pp. 389-408, 2009.

[14] R. T. Rockafellar, Convex Analysis, Princeton Mathematical Series, No. 28, Princeton University Press, Princeton, NJ, USA, 1970.

[15] Y. P. Fang, K. Meng, and X. Q. Yang, "Piecewise linear multicriteria programs: the continuous case and its discontinuous generalization," Operations Research, vol. 60, no. 2, pp. 398-409, 2012.

[16] X. H. Gong, "Connectedness of the efficient solution set of a convex vector optimization in normed spaces," Nonlinear Analysis: Theory, Methods \& Applications, vol. 23, no. 9, pp. 11051114, 1994.

[17] D. T. Luc, "Connectedness of the efficient point sets in quasiconcave vector maximization," Journal of Mathematical Analysis and Applications, vol. 122, no. 2, pp. 346-354, 1987.

[18] E. K. Makarov and N. N. Rachkovski, "Efficient sets of convex compacta are arcwise connected," Journal of Optimization Theory and Applications, vol. 110, no. 1, pp. 159-172, 2001.

[19] E. J. Sun, "On the connectedness of the efficient set for strictly quasiconvex vector minimization problems," Journal of Optimization Theory and Applications, vol. 89, no. 2, pp. 475481, 1996.

[20] X. Y. Zheng, "Contractibility and connectedness of efficient point sets," Journal of Optimization Theory and Applications, vol. 104, no. 3, pp. 717-737, 2000.

[21] J. Jahn, Vector Optimization: Theory, Applications, and Extensions, Springer, Berlin, Germany, 2004.

[22] D. T. Luc, "Contractibility of efficient point sets in normed spaces," Nonlinear Analysis: Theory, Methods \& Applications, vol. 15 , no. 6, pp. 527-535, 1990.

[23] A. R. Warburton, "Quasiconcave vector maximization: connectedness of the sets of Pareto-optimal and weak Paretooptimal alternatives," Journal of Optimization Theory and Applications, vol. 40, no. 4, pp. 537-557, 1983. 


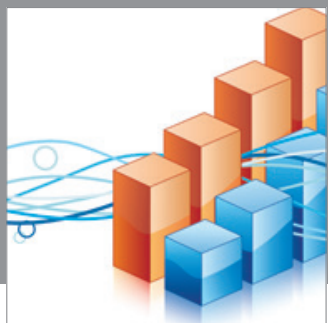

Advances in

Operations Research

mansans

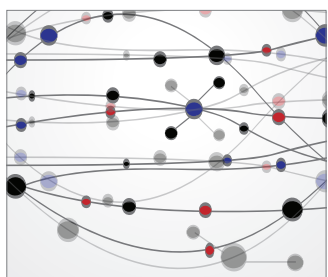

The Scientific World Journal
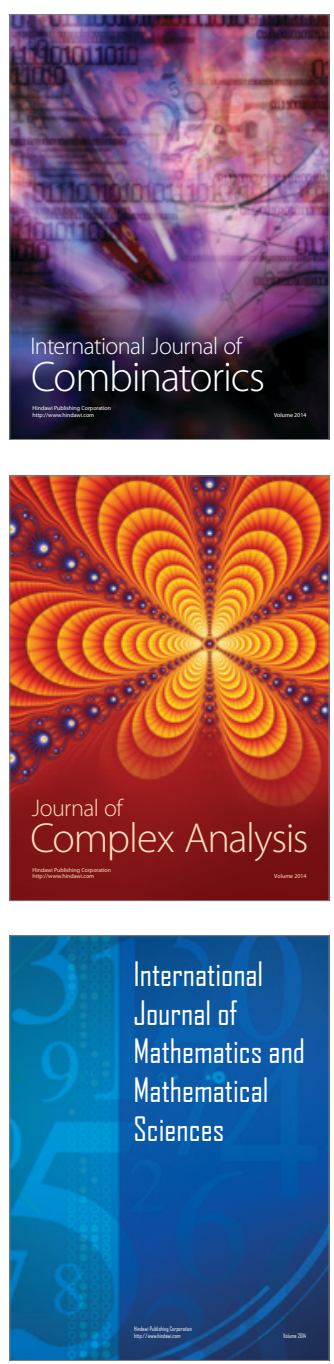
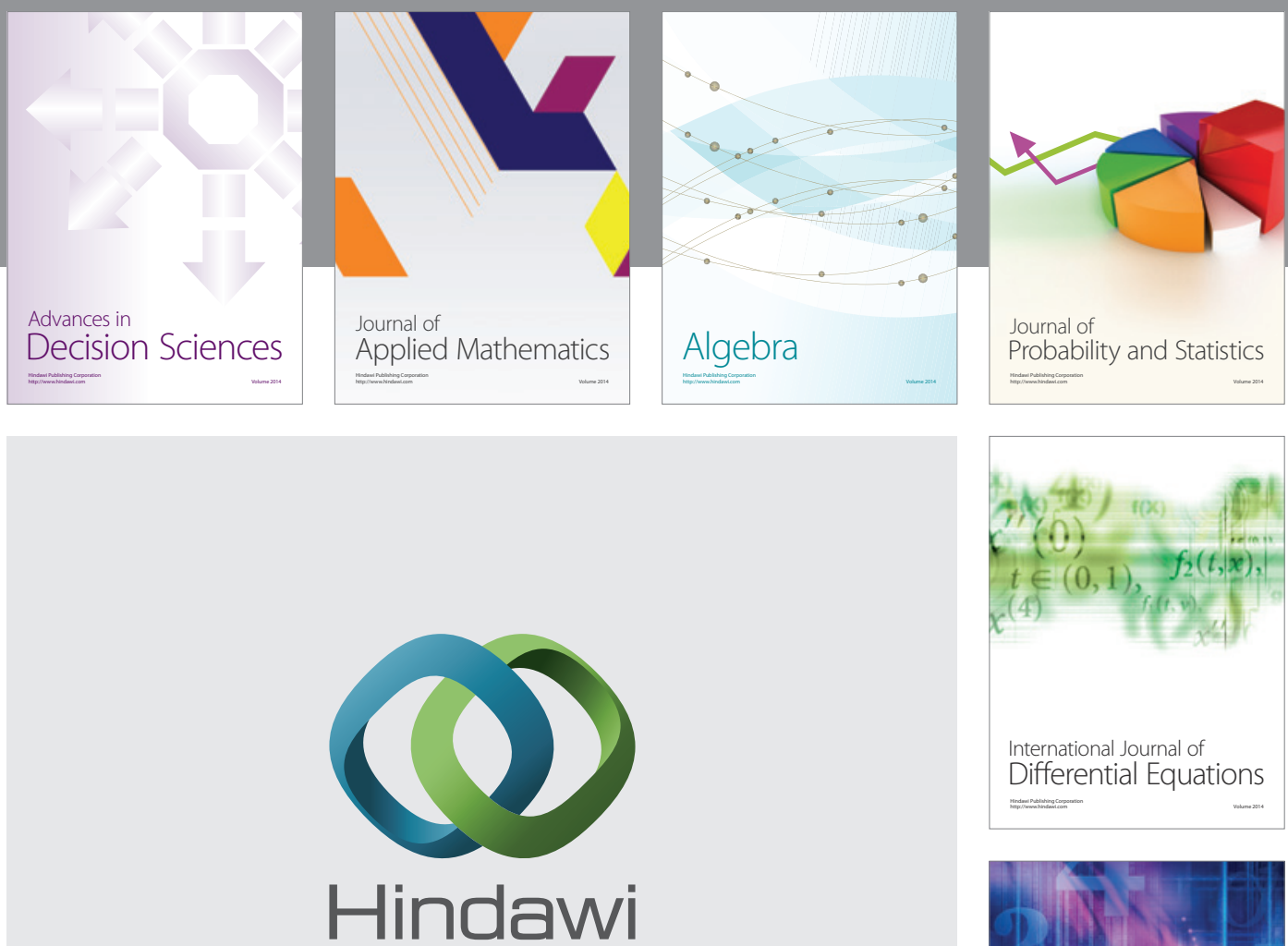

Submit your manuscripts at http://www.hindawi.com
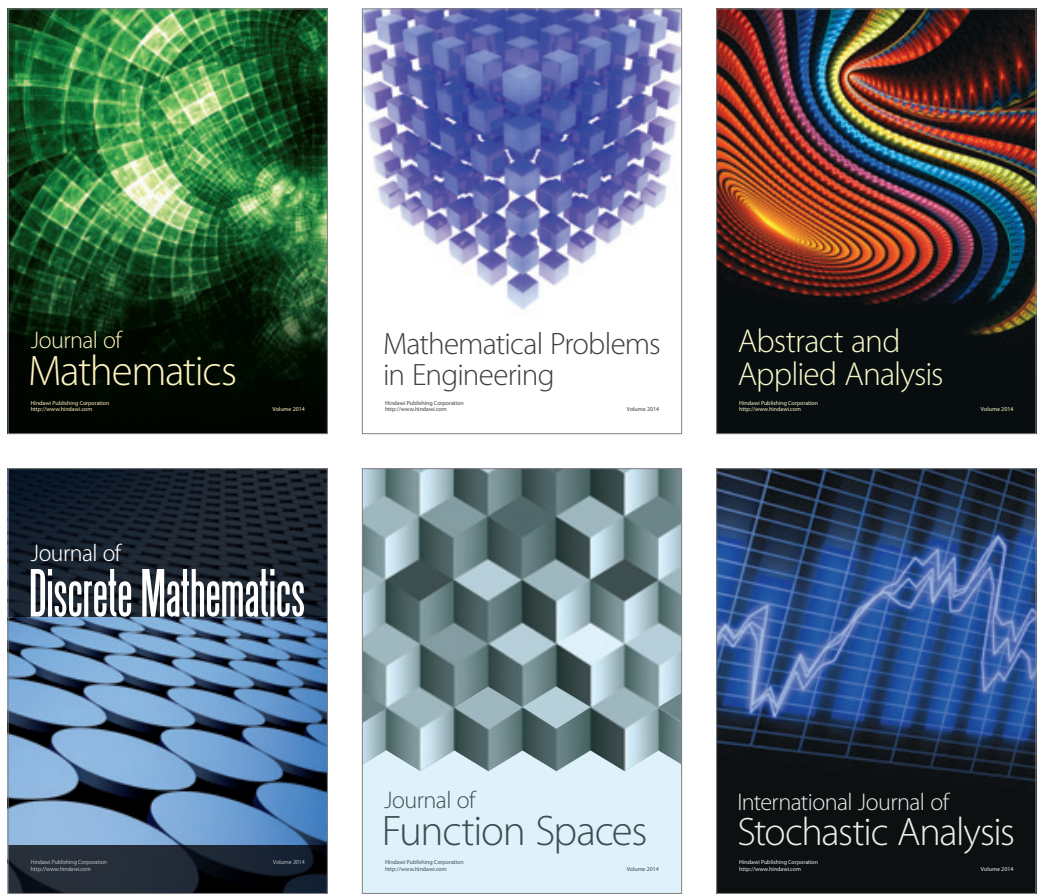

Journal of

Function Spaces

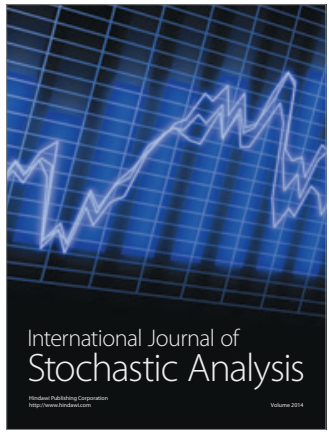

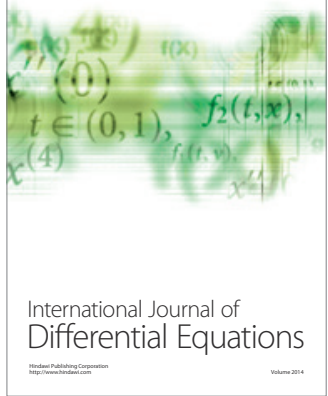
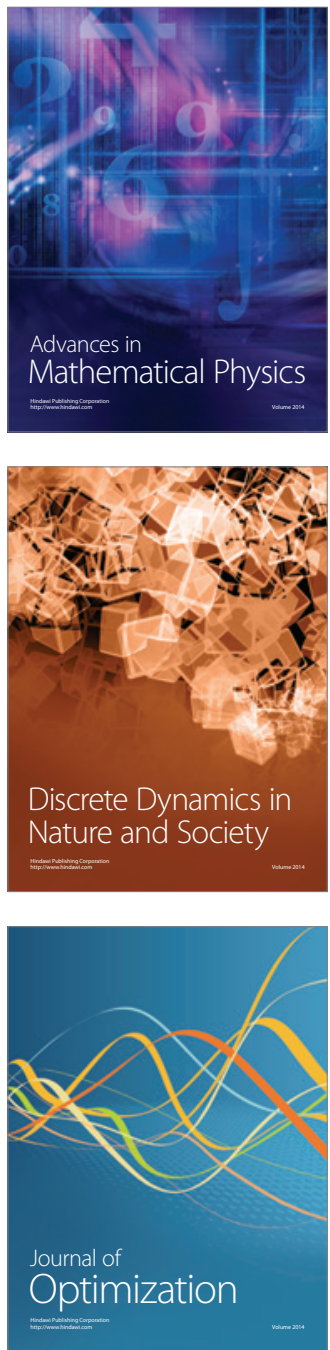